\title{
Booksight: Visualising the Library of Ideas
}

\author{
Mel Woods \\ University of Dundee \\ Perth Road, Dundee, DD1 4HT \\ m.j.woods@dundee.ac.uk
}

\author{
Hadi Mehrpouya \\ University of Dundee \\ Perth Road, Dundee, DD1 4HT \\ h.mehrpouya@dundee.ac.uk
}

\author{
Jamie Forth \\ Queen Mary University of London \\ Mile End Road London E1 4NS \\ jamie.forth@eecs.qmul.ac.uk
}

\section{INTRODUCTION}

As libraries extend their reach beyond the constraint of physical fixed space via the fast and efficient use of online and digital resources, (Sadler 2012) a new paradigm for tools to support the role of the library in the digital age are being considered (Frischer 2005). The special architecture of the physical library has been designed specifically to enable information search and discovery through a transparent classification system and organisation, as well as facilitation of visual browsing. In this sense the library could be seen is an act of inspiration architecture, it enables search, and at the same time physically reminds us the path to discovery doesn't usually take a straight line. However, the digital library offers no such physical or visual feedback, and as libraries themselves move towards blended or fully digital collections this may affect users experience and engagement with the archive or collection and awareness of personal pathways as well as the activity of others' within the library space itself.

The SerenA research project seeks to understand serendipity and its role in research (Makri 2011), in order to facilitate it through the design of digital tools for personal research (Maxwell 2012) and future research spaces. The role of serendipity and its association with finding things you didn't know you needed to know has been attributed to scientific, it is widely acknowledged as a valuable phenomenon across disciplines, within business and industry and to those dedicated to the pursuit of knowledge, discovery and research.

We propose real time book requests in a library as one way to investigate the visualisation of data to support serendipity. 'Booksight' library information visualisation is designed to curate the real-time flow of book requests which represent the titles and authors of books, in a way that offers the viewer potentially surprising and valuable connections between words, concepts and images. Firstly, we seek to make visible human digital research, by making manifest anonymous research. Secondly, we present a new space for browsing digital books in a physical space in a similar way to the returns shelf. Thirdly, we propose to assess support for the occurrence of surprising and valuable information discovery.

\section{LIBRARY DATA}

User interactions with digital library collections such as digital search and request for books, both within the library and increasingly remotely through online library portals, offers a dataset that has the potential to be enriched and displayed into the library environment. 'Booksight' uses a backend system that receives data of book requests direct from the Library system. A goal of the system is not only to present the data itself, but enrich it.

\section{PROTOTYPE DEVELOPMENT}

The prototype 'Booksight' is conceived in the context of a major UK city library, and designed to be projected on to the walls of the building itself. $A$ study conducted at The British Library outlined the design context and embodied public spaces, paying particular attention to user activities, traffic, flow, noise and light levels. Design guidelines to support serendipity (Maxwell 2012) such as the concepts of reflexivity, playfulness, ambiguity and delight were employed through prototypes for a number of designs each of which were evaluated.

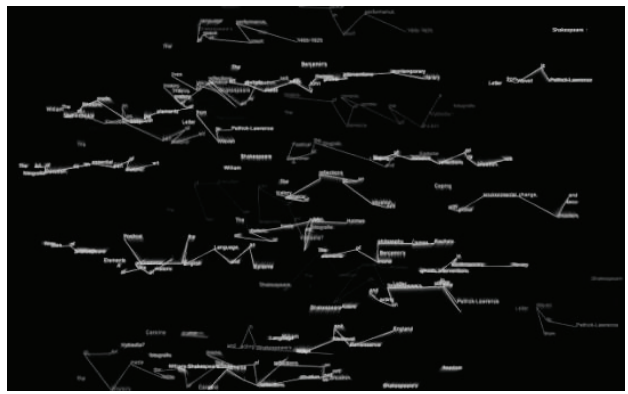

Figure 1: Individual words from book titles and authors, broken down, remixed with linguistic style matching

Early prototype examples included 1) a simple waterfall stream of book titles and authors, and; 2) a branch and tree formation representation of new 
associations (Fig 1). The final visualisation (Fig 2) employs a backend system to enrich the data by linking catalogue requests to original book covers, some of which are no longer available in the library itself. Animation of the book covers is driven by a bespoke physics engine, allowing user interaction via gesture (using Wii mote), or presentation as an ambient stream of visual images. The visualisation is reminiscent of browsing the physical bookshelf through digital representation of live book requests in the physical environment.

\section{SYSTEM DEVELOPMENT}

From a technological perspective, SerenA is a Semantic Web-based, distributed, multi-agent system. The architecture enables integration and supports a number of user interactions of which Booksight is one. Three core technologies underlie the SerenA development: 1) the Resource Description Framework (RDF, http://www.w3.org/RDF/), and associated developments of the Semantic Web and Linked Open Data; 2) Jade (http://jade.tilab.com/), a mature Java-based agent programming framework; and 3) WebSockets (http://tools.ieff.org/html//fc6455), enabling bi-directional real-time communication between SerenA agents, private and public instantiations over the Web.

Booksight utilises the backend infrastructure primarily to clean, transform, and enrich structured bibliographic metadata, including author names and item titles, that represent requests from the British Library catalogue. Specialised agents within the system then retrieve further information about each requested item (e.g. images) from sources on the Web, as well as carry out simple natural language processing of textual data providing annotations of linguistic constructs (e.g. parts of speech tags) and semantic identifiers (e.g. named entity URIs for people, places and concepts). The enriched data, now in RDF, is published to a secure private WebSocket channel, to which the frontend processing visualisation software subscribes to this system as well as responding to the live stream the frontend can also query the backend for specific information to integrate into the visualisation.

\section{CONCLUSIONS AND NEXT STEPS}

Iterative and agile development, continuous testing and evolution of 'Booksight' created a range of design considerations and practice based outcomes to support data visualisation within a library context. However, by embedding the visualisation in contexts, such as a research centre, board-room, gallery space and meeting space, we discovered that participants recognised real-time representation of information as a link into the flow of ideas and people elsewhere. Next steps include a longitudinal evaluation, in order to explore whether Booksight supports valuable and surprising connections that we might consider as serendipitous. Future work will focus on opportunities for library catalogue enrichment using open data, to engage and enhance learning, teaching and research activities and spaces.

\section{REFERENCES}

Frischer, B (2005) The Ultimate Internet Café: Reflections of a Practicing Digital Humanist about Designing a Future for the Research Library in the Digital Age. Library as Place: Rethinking Roles, Rethinking Space. Council on Library and Information Resources. Canada.

Makri, S., and Blandford, A. (2011) What is Serendipity? A Workshop Report. In Thematic Issue of Information Research on the Opportunistic Discovery of Information, 16(3), September 2011.

Maxwell, D., Woods, M., Makri, S., Bental, D., Sharples, S., and Kefalidou, G. (2012) Designing a Semantic Sketchbook to Create Opportunities for Serendipity. BCS HCl Conference 2012, University of Birmingham, 12-14 Sept. 2012. ACM Digital Library.

Maxwell, D., and Woods, M. (2012) Encouraging Reflexivity in Mobile Interactions Accepted for CHArt 28TH Annual Conference, London.

Sadler, B. (2012) Brain Injuries, Science Fiction, and Library Discovery. http://accessconference.ca (retrieved $17^{\text {th }}$ January 2013).
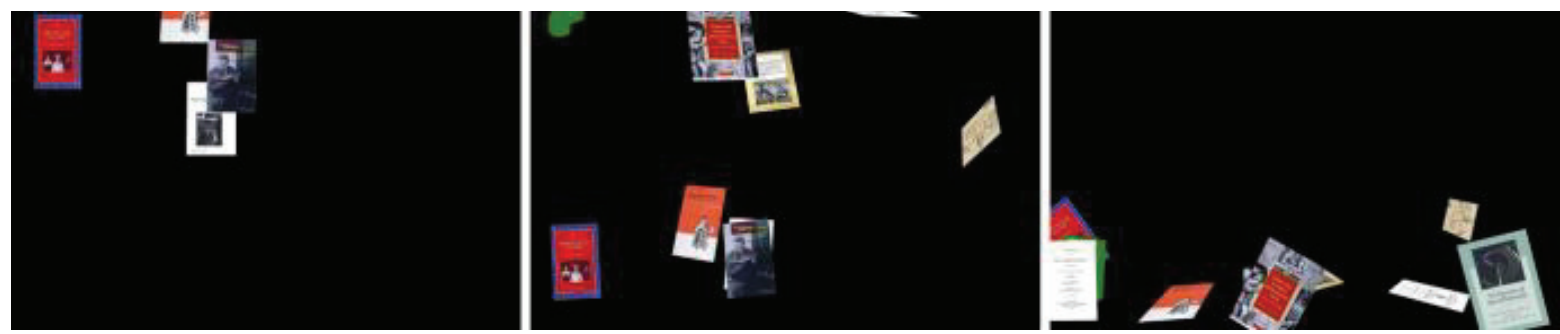

Figure 2: Real time catalogue requests connected to external sources to represent by visual falling book covers 